Real Analysis Exchange

Vol. 23(2), 1997-1998, pp. 757-766

A. K. Layek*, Department of Mathematics, Bangabasi College of Commerce, 35, Scott Lane, Calcutta 700009, West Bengal, India e-mail:

ssg@cal . vsnl . net . in

\title{
INTERSECTION PROPERTIES OF DIRECTIONAL ESSENTIAL CLUSTER SETS
}

\begin{abstract}
Jarnik [3] in 1936 proved a remarkable property of directional cluster sets. This result states that for a function $f$ defined on the open upper half plane to the extended real line, each pair of directional cluster sets intersect at all points on the real line but a countable set of points. In [5] an example was constructed to show that the exact analogue of Jarnik's result fails for directional essential cluster sets. Here we shall establish a certain variant of this analogue for directional essential cluster sets of measurable functions. The result which will be proved here is also related with the results in Theorem 2 of [1] and in Theorem 2 of [2].
\end{abstract}

\section{Preliminaries}

Let $H, \mathbb{R}, M^{*}(A)$ and $\bar{A}$ denote, respectively, the open upper half plane, the real line, Lebesgue outer measure of the set $A$, and the closure of the set $A$. The Lebesgue outer measure is linear or planar and which will be clear from the context.

For $x \in \mathbb{R}, h>0$ and $\theta \in(0, \pi)$ we set

$$
\begin{gathered}
K(x, h)=\{z: z \in H,|z-x|<h\}, \\
L_{\theta}(x)=\{z: z \in H, \arg (z-x)=\theta\}
\end{gathered}
$$

and

$$
L_{\theta}(x, h)=L_{\theta}(x) \cap K(x, h) .
$$

Key Words: Lebesgue outer measure, densities, countable, directional cluster sets, directional essential cluster sets

Mathematical Reviews subject classification: 30D40

Received by the editors May 9, 1997

*I am thankful to the referee for his kind suggestions on the style of the paper 
For each pair of directions, $u$ and $v, 0<u<v<\pi$, let $S_{u v}$ be a sector in $H$ with vertex at the origin, defined by

$$
S_{u v}=\{z: z \in H, u<\arg (z)<v\} .
$$

By $S_{u v}(x)$ we mean the translate of $S_{u v}$ which is obtained by taking the origin at $x$. If there is no confusion we shall simply write $S$ and $S(x)$ for the sets $S_{u v}$ and $S_{u v}(x)$. Further set

$$
S(x, h)=S(x) \cap K(x, h) .
$$

The sectorial upper outer density $\bar{d}^{*}(E, x, S)$ and the sectorial outer density $d^{*}(E, x, S)$ of $E \subset H$ at $x \in \mathbb{R}$ are respectively defined as

$$
\bar{d}^{*}(E, x, S)=\limsup _{h \rightarrow 0} \frac{M^{*}(E \cap S(x, h))}{M^{*}(S(x, h))}
$$

and

$$
d^{*}(E, x, S)=\lim _{h \rightarrow 0} \frac{M^{*}(E \cap S(x, h))}{M^{*}(S(x, h))} .
$$

The directional outer densities $\bar{d}^{*}(E, x, \theta)$ and $d^{*}(E, x, \theta)$ of $E \subset H$ at $x \in \mathbb{R}$ and in the direction $\theta \in(0, \pi)$ can be defined as sectorial outer densities by replacing $S(x, h)$ by $L_{\theta}(x, h)$ and considering the linear outer measure.

Whenever the sets concerned are measurable then we drop the word "outer" and the mark "*".

Let $f: H \rightarrow W$, where $W$ is a topological space. The directional essential cluster set $C_{e}(f, x, \theta)$ of $f$ at $x$ in the direction $\theta \in(0, \pi)$ is the set of all $w$ in $W$ such that for every open set $U$ of $W$ containing $w, \bar{d}^{*}\left(f^{-1}(U), x, \theta\right)>0$. The directional cluster set $C(f, x, \theta)$ can be defined as $C_{e}(f, x, \theta)$, but $\bar{d}^{*}(E, x, \theta)>$ 0 is to be replaced by

$$
L_{\theta}(x, r) \cap f^{-1}(U) \neq \emptyset \text { for all } r>0 .
$$

A set $E \subset H$ is said to have Baire property if $E=G \Delta Q$, where $G$ is an open set and $Q$ is a first category set in $H$. If, in particular $Q$ is a countable set then the set $E$ is said to have restricted Baire property.

A function $f: H \rightarrow W$, where $W$ is a topological space, is said to have Baire (resp. restricted Baire) property if for every open set $U$ in $W, f^{-1}(U)$ has Baire (resp. restricted Baire) property. 
2

Let $E \subset H$ be measurable. For $x \in \mathbb{R}$ set

$$
\begin{gathered}
\Pi(E, x)=\{\theta: 0<\theta<\pi, d(E, x, \theta)=0\} \\
\mathcal{V}(E, x)=\{\theta: 0<\theta<\pi, d(E, x, \theta)=1\},
\end{gathered}
$$

and for $\phi<\varphi$ in $(0, \pi), \mathcal{V}_{\phi \varphi}(E, x)=\mathcal{V}(E, x) \cap(\phi, \varphi)$.

For positive integer $m$, positive and fixed real $K<1$, and rationals $\alpha, \beta$, $\gamma$ and $\delta$ in $(0, \pi)$ with $\alpha<\beta, \gamma<\delta$ and $(\alpha, \beta) \subset(0, \pi) \backslash[\gamma, \delta]$ let

$$
\begin{gathered}
\mathcal{V}_{\alpha \beta m}(E, x, \gamma, \delta)= \\
=\left\{\theta: \theta \in(\alpha, \beta), \quad M\left(E \cap L_{\theta}(x, h)\right)>K h, \text { for all } h, 0<h<\frac{1}{m}\right\} .
\end{gathered}
$$

Then clearly for all $\alpha_{1}$ and $\beta_{1}$ with $\alpha_{1}<\beta_{1}$ in $(0, \pi) \backslash[\gamma, \delta]$

$$
\mathcal{V}_{\alpha_{1} \beta_{1}}(E, x) \subset \cup_{\alpha} \cup_{\beta} \cup_{m} \mathcal{V}_{\alpha \beta m}(E, x, \gamma, \delta), \quad \alpha_{1}<\alpha<\beta<\beta_{1} .
$$

These sets and relation will be used in the sequel. The density function $d(E, x, \theta)$ is measurable [6, Lemma 2], so the sets $\Pi(E, x)$ and $\mathcal{V}(E, x)$ are measurable. It can also be shown that the sets $\mathcal{V}_{\alpha \beta m}(E, x, \gamma, \delta)$ are also measurable (see Lemma 1 in $[6]$ ).

Lemma 1. If $E \subset H$ is measurable then the set

$$
T(E)=\{x: x \in \mathbb{R}, M \Pi(E, x)>0 \text { and } M \mathcal{V}(E, x)>0\}
$$

is countable.

Proof. For rationals $\alpha, \beta, \gamma, \delta, \mu$ and $\nu$ with $0<\alpha<\beta<\gamma<\delta<\mu<\nu<\pi$ and positive integers $m, n$ and rationals $p$ and $q$ in $(0,1)$ set

$T_{\alpha \beta \gamma \delta m p}=\left\{x: x \in \mathbb{R}, M(\Pi(E, x) \cap(\gamma, \delta))>0\right.$ and $\left.M \mathcal{V}_{\alpha \beta m}(E, x, \gamma, \delta)>p\right\}$

and $T_{\mu \nu \gamma \delta n q}$ be a similar set and it is obtained by replacing $\alpha, \beta, m$ and $p$ with $\mu, \nu, n$ and $q$. Then using the relation (A) it can be shown that

$$
T(E) \subset \cup\left(T_{\alpha \beta \gamma \delta m p} \cup T_{\mu \nu \gamma \delta n q}\right),
$$

where the union is taken for all rationals $\alpha, \beta, \gamma, \delta, \mu$ and $\nu$ in $(0, \pi)$ with $\alpha<\beta<\gamma<\delta<\mu<\nu$, for all positive integers $m$ and $n$ and all rationals $p$ 
and $q$ in $(0,1)$. For fixed $\alpha, \beta, \gamma, \delta, m$ and $p$ let $T=T_{\alpha \beta \gamma \delta m p}$. Suppose $x^{\prime} \in T$ is a left limit point of $T$. Let $\left\{x_{k}\right\} \subset T$ be a sequence such that $x_{k}<x_{k+1}<x^{\prime}$ for all $k$ and $\lim _{k \rightarrow \infty} x_{k}=x^{\prime}$. Suppose $k_{1}$ is the first integer so that

$$
S_{\alpha \beta}\left(x_{k}, \frac{1}{m}\right) \cap S_{\gamma \delta}\left(x^{\prime}\right)
$$

is a quadrilateral for all $k \geq k_{1}$. Let $u$ and $v$ be such that $\gamma<u<v<\delta$ and $(v-u)<\pi / 3$. Then for all $k \geq k_{1}$,

$$
S_{\alpha \beta}\left(x_{k}, \frac{1}{m}\right) \cap S_{u v}\left(x^{\prime}\right)
$$

is also a quadrilateral, say it $Q_{k}$. Let $k \geq k_{1}$ be fixed and $x_{k}=x_{0}$. For $\theta \in$ $(\alpha, \beta)$ set $I_{\theta}=Q_{0} \cap L_{\theta}\left(x_{0}\right)$. Then $I_{\theta} \cap E$ is measurable for $\theta \in \mathcal{V}_{\alpha \beta m}\left(E, x_{0}, \gamma, \delta\right)$ $=D$ (say). Henceforth, for convenience, we shall simply write $I, Q$ and $x$ for $I_{\theta}, Q_{0}$ and $x_{0}$, respectively. For $\theta \in D$ and $\phi \in[u, v]$ let

$$
r_{\theta \phi}=\left|L_{\theta}(x) \cap L_{\phi}\left(x^{\prime}\right)-x\right|,
$$

i.e. $r_{\theta \phi}$ is the distance between $x$ and $L_{\theta}(x) \cap L_{\phi}\left(x^{\prime}\right)$. Now it can be shown that

$$
r_{\theta v}=\frac{r_{\theta u} \sin (u-\theta) \sin v}{\sin (v-\theta) \sin u} .
$$

Then we get

$$
M(Q \cap E) \geq \int_{D} \int_{I \cap E} r d r d \theta
$$

Further we have

$$
\int_{I \cap E} r d r>r_{\theta v} M(I \cap E)=r_{\theta v}\left|M\left(L_{\theta}\left(x, r_{\theta u}\right) \cap E\right)-M\left(L_{\theta}\left(x, r_{\theta v}\right) \cap E\right)\right| .
$$

Hence $r_{\theta v} M(I \cap E)>\left|P r_{\theta u}-P r_{\theta v}\right| \cdot r_{\theta v}$, where $P$ is the minimum of

$$
\frac{M\left(L_{\theta}\left(x, r_{\theta u}\right) \cap E\right)}{r_{\theta u}} \text { and } \frac{M\left(L_{\theta}\left(x, r_{\theta v}\right) \cap E\right)}{r_{\theta v}} .
$$

Now using (2) we get

$$
r_{\theta v} \cdot M(I \cap E)>r_{\theta v} \cdot r_{\theta u} \cdot P\left(\frac{\sin \theta \sin (v-u)}{\sin (v-\theta) \sin u}\right) .
$$

Since $\sin \theta>\sin \alpha \cdot \sin \beta, 0<\sin (v-\theta) \sin u<1$ and $P \geq K$, so we get after simple calculations

$$
M(Q \cap E)>K \cdot \sin (v-u) \cdot \sin \alpha \cdot \sin \beta \cdot\left(x^{\prime}-x\right)^{2} \int_{D} \frac{\sin u \cdot \sin v}{\sin (v-\theta) \sin (u-\theta)} d \theta .
$$


Again since

$$
0<\sin (v-\theta) \cdot \sin (u-\theta)<1 \quad \text { and } \quad \sin u \cdot \sin v>\sin ^{2} \gamma \cdot \sin ^{2} \delta,
$$

setting

$$
h_{0}=\max \left\{\frac{\sin \beta}{\sin (v-\beta)}, \frac{\sin \beta}{\sin (u-\beta)}\right\} \cdot\left(x^{\prime}-x\right),
$$

we get

$$
M(Q \cap E)>h_{0}^{2} \cdot \sin (v-u) \cdot p \cdot K \cdot \sin \alpha \cdot \sin ^{2} \gamma \cdot \sin ^{2} \delta \cdot \sin ^{2}(\varphi-\beta),
$$

where $\varphi=v$ or $u$ according to the requirement. Now, since $\sin (\varphi-\beta)>$ $\sin (\gamma-\beta) \cdot \sin (\delta-\beta)$ and $2 \sin \theta>\theta$ for $0<\theta<\pi / 3$, we get from (B)

$$
M\left(S_{u v}\left(x^{\prime}, h_{0}\right) \cap E\right)>M\left(S_{u v}\left(x^{\prime}, h_{0}\right)\right) \cdot \hat{k},
$$

where

$$
\hat{k}=K \cdot \sin \alpha \cdot \sin ^{2} \gamma \cdot \sin ^{2} \delta \cdot \sin ^{2}(\gamma-\beta) \cdot \sin ^{2}(\delta-\beta) \cdot p .
$$

Thus one gets

$$
\frac{M\left(S_{u v}\left(x^{\prime}, h_{0}\right) \cap E\right)}{M\left(S_{u v}\left(x^{\prime}, h_{0}\right)\right)}>\hat{k}, \quad 0<\hat{k}<1 .
$$

Since (3) is true for all $x_{k}\left(=x_{0}\right)$ for $k \geq k_{1}$ and since $h_{k} \rightarrow 0$ as $x_{k} \rightarrow x^{\prime}$, so taking limit as $x_{k} \rightarrow x^{\prime}$ ultimately we get

$$
\begin{gathered}
\qquad \bar{d}\left(E, x^{\prime}, S_{u v}\right) \geq \hat{k} \\
\text { for all } u \text { and } v \text { with } v-u<\frac{\pi}{3} \text { and } 0<\gamma<u<v<\delta<\pi .
\end{gathered}
$$

For positive integer $t$ let

$$
\begin{gathered}
\Pi_{t}\left(E, x^{\prime}, \gamma, \delta\right)= \\
=\left\{\theta: \gamma<\theta<\delta, \quad M\left(L_{\theta}\left(x^{\prime}, h\right) \cap E\right)<\hat{k} \cdot \frac{h}{2} \text { for all } h, \quad 0<h<\frac{1}{t}\right\} .
\end{gathered}
$$

Then clearly,

$$
\Pi\left(E, x^{\prime}\right) \cap(\gamma, \delta) \subset \cup_{t=1}^{\infty} \Pi_{t}\left(E, x^{\prime}, \gamma, \delta\right) .
$$

Since $M\left(\Pi\left(x^{\prime}\right) \cap(\gamma, \delta)\right)>0$ so there is a $t$ such that $M\left(\Pi_{t}\left(E, x^{\prime}, \gamma, \delta\right)\right)>0$. Let $\varphi$ be a point of density of $\Pi_{t}\left(E, x^{\prime}, \gamma, \delta\right)$. Then for $\epsilon, 0<\epsilon<\hat{k}^{2} / 4$ there is $\eta, 0<\eta<\pi / 6$, such that for all $r, 0<r<\eta$ and $\gamma<\varphi-r<\varphi+r<\delta$,

$$
M\left(\Pi_{t}\left(E, x^{\prime}, \gamma, \delta\right) \cap(\varphi-r, \varphi+r)\right)>2 r(1-\epsilon) .
$$


Let $r=r_{0}<\eta<\pi / 6$ be fixed and let $\varphi-r_{0}=i>\gamma$ and $\varphi+r_{0}=j<\delta$. Then for $h, 0<h<1 / t$ and writing $(i, j)=\Pi$ and $\Pi_{t}\left(E, x^{\prime}, \gamma, \delta\right)=\mathcal{V}$ we get

$$
\begin{gathered}
M\left(S_{i j}\left(x^{\prime}, h\right) \cap E\right)= \\
\frac{1}{2} \int_{\Pi \cap \mathcal{V}}\left(M\left(L_{\theta}\left(x^{\prime}, h\right) \cap E\right)\right)^{2} d \theta+\frac{1}{2} \int_{\Pi \backslash \mathcal{V}}\left(M\left(L_{\theta}\left(x^{\prime}, h\right) \cap E\right)\right)^{2} d \theta< \\
<\frac{1}{2}\left(h^{2} \cdot \frac{\hat{k}^{2}}{4} \cdot M \Pi+h^{2} \cdot \epsilon \cdot 2 r_{0}\right)<M\left(S_{i j}\left(x^{\prime}, h\right)\right) \cdot \frac{\hat{k}^{2}}{2},
\end{gathered}
$$

using (5), and $2 r_{0}=M \Pi$ and $\epsilon<\hat{k}^{2} / 4$. We get

$$
\frac{M\left(S_{i j}\left(x^{\prime}, h\right) \cap E\right)}{M S_{i j}\left(x^{\prime}, h\right)}<\frac{\hat{k}^{2}}{2} .
$$

Taking limit as $h \rightarrow 0$, finally we get

$$
\bar{d}\left(E, x^{\prime}, S_{i j}\right)<\frac{\hat{k}^{2}}{2} .
$$

Since $\gamma<i<j<\delta$ and $(j-i)<\pi / 3$, so (4) and (6) are contradictory and hence the set $T=T_{\alpha \beta \gamma \delta m p}$ is countable for all rationals $0<\alpha<\beta<\gamma<$ $\delta<\pi$, all integers $m$ and for all rationals $p$ in $(0,1)$. By approaching from the right it can be shown as above that no point of $T_{\mu \nu \gamma \delta n q}$ is a right limit point of it. Thus, the set $T(E)$ in (1) is countable. This completes the proof.

Lemma 2. If $E \subset H$ is open then the set $K(E)=\{x: x \in \mathbb{R}, \Pi(E, x)$ and $\mathcal{V}(E, x)$ are second category sets in $(0, \pi)\}$ is countable.

Proof. For rationals $\alpha, \beta, \gamma, \delta, \mu$ and $\nu$ in $(0, \pi)$ with $\alpha<\beta<\gamma<\delta<\mu<\nu$, and positive integers $m$ and $n$ let $K_{\alpha \beta \gamma \delta m}=\{x: x \in \mathbb{R}, \Pi(E, x) \cap(\gamma, \delta)$ is a second category set and $\mathcal{V}_{\alpha \beta m}(E, x, \gamma, \delta)$ is dense in $\left.(\alpha, \beta)\right\}$, and $K_{\mu \nu \gamma \delta n}$ be a similar set but $\alpha, \beta$ and $m$ are replaced by $\mu, \nu$ and $n$ respectively. Then by using the relation (A), it can be shown that

$$
K(E) \subset \cup\left(K_{\alpha \beta \gamma \delta m} \cup K_{\mu \nu \gamma \delta n}\right),
$$

where the union is taken for all rationals $\alpha<\beta<\gamma<\delta<\mu<\nu$ in $(0, \pi)$ and positive integers $m$ and $n$.

For $x \in K_{\alpha \beta \gamma \delta m}$, the facts $\mathcal{V}_{\alpha \beta m}(\bar{E}, x, \gamma, \delta)$ is dense in $(\alpha, \beta)$ and $\bar{E}$ is a closed set, imply that for every $\theta \in(\alpha, \beta), M\left(L_{\theta}(x, h) \cap \bar{E}\right) \geq K h$ for all $h$, $0<h<1 / m$. Let $x^{\prime} \in K_{\alpha \beta \gamma \delta m}$ be a left limit point of the set $K_{\alpha \beta \gamma \delta m}$. 
Then just replacing the sets $E$ and $D=\mathcal{V}_{\alpha \beta m}\left(E, x_{0}, \gamma, \delta\right)$ by the sets $\bar{E}$ and $(\alpha, \beta)$ throughout the first part of the proof of Lemma 1, we can get a real $k$, $0<k<1$, analogous to $\hat{k}$ such that

$$
\bar{d}\left(\bar{E}, x^{\prime}, S_{u v}\right) \geq k,
$$

for all $u, v$ with $\gamma<u<v<\delta$ and $(v-u)<\pi / 3$.

Defining the set $\Pi_{t}\left(E, x^{\prime}, \gamma, \delta\right)$ as in the second part of the proof of Lemma 1 replacing $\hat{k}$ by $k$ we get

$$
\Pi\left(E, x^{\prime}\right) \cap(\gamma, \delta) \subset \cup_{t=1}^{\infty} \Pi_{t}\left(E, x^{\prime}, \gamma, \delta\right) .
$$

As $\Pi\left(E, x^{\prime}\right) \cap(\gamma, \delta)$ is a second category set, there is a positive integer $t=p$ so that $\Pi=\Pi_{p}\left(E, x^{\prime}, \gamma, \delta\right)$ is dense in an open interval $(\phi, \varphi) \subset(\gamma, \delta)$ with $\varphi-\phi<\pi / 3$. Since $E$ is open and $\Pi$ is dense in $(\phi, \varphi)$, so for every $\theta \in(\phi, \varphi)$,

$$
M\left(L_{\theta}\left(x^{\prime}, h\right) \cap E\right) \leq k \frac{h}{2}, \quad \text { for all } h, \quad 0<h<\frac{1}{p} .
$$

Thus, for $0<h<\frac{1}{p}$, we have

$$
M\left(S_{\phi \varphi}\left(x^{\prime}, h\right) \cap E\right)=\int_{\phi}^{\varphi} \frac{1}{2}\left(M\left(L_{\theta}\left(x^{\prime}, h\right) \cap E\right)\right)^{2} d \theta<\frac{1}{2} k^{2} h^{2}(\varphi-\phi),
$$

i.e.,

$$
M\left(S_{\phi \varphi}\left(x^{\prime}, h\right) \cap E\right)<k^{2} \cdot M\left(S_{\phi \varphi}\left(x^{\prime}, h\right)\right) .
$$

Since for bounded open set $F \subset H, M(F)=M(\bar{F})$, we get

$$
M\left(S_{\phi \varphi}\left(x^{\prime}, h\right) \cap E\right)=M\left(\overline{S_{\phi \varphi}\left(x^{\prime}, h\right) \cap E}\right)=M\left(S_{\phi \varphi}\left(x^{\prime}, h\right) \cap \bar{E}\right) .
$$

Thus we get from above

$$
\frac{M\left(S_{\phi \varphi}\left(x^{\prime}, h\right) \cap \bar{E}\right)}{M S_{\phi \varphi}\left(x^{\prime}, h\right)}<k^{2} .
$$

Taking limit as $h \rightarrow \infty$ we ultimately get

$$
\bar{d}\left(\bar{E}, x^{\prime}, S_{\phi \varphi}\right) \leq k^{2} .
$$

Since (2) and (3) are contradictory, each set $K_{\alpha \beta \gamma \delta m}$ is countable.

Proceeding from the right it can be similarly shown that no point of $K_{\mu \nu \gamma \delta n}$ is a right limit point of it. Thus, the set $K(E)$ is countable. 
Lemma 2.1. If $E \subset H$ is open then the set $T^{\prime}(E)=\{x: x \in \mathbb{R}, M \Pi(E, x)$ $>0$ and $\mathcal{V}(E, x)$ is second category in $(0, \pi)\}$ is countable.

Lemma 2.2. If $E \subset H$ is open then the set $K^{\prime}(E)=\{x: x \in \mathbb{R}, \Pi(E, x)$ is second category in $(0, \pi)$ and $M \mathcal{V}(E, x)>0\}$ is countable.

The proofs are quite similar to that of Lemma 2, so we have omitted them.

Lemma 3. Let $F: H \rightarrow W$ be arbitrary, where $W$ is a compact topological space. If $G$ is an open set and $F$ is a closed set in $W$ such that $C_{e}(f, x, \theta) \subset G$ and $C_{e}(f, x, \phi) \cap F=\emptyset$ then

$$
d^{*}\left(f^{-1}(G), x, \theta\right)=1 \quad \text { and } \quad d^{*}\left(f^{-1}(F), x, \phi\right)=0 .
$$

Proof. The first part is proved in Lemma 2 of [4] and the proof of the second part is similar.

Theorem. Let $f: H \rightarrow W$ be measurable, where $W$ is a compact, normal and second countable topological space. Then except possibly at most a countable set of points $x \in \mathbb{R}$, for almost every $\theta$ in $(0, \pi)$

$$
C_{e}(f, x, \theta) \cap C_{e}(f, x, \phi) \neq \emptyset
$$

for almost every $\phi \in(0, \pi)$.

If further $f$ is continuous then except possibly at most a countable set of points $x \in \mathbb{R}$, for almost every and nearly every $\theta \in(0, \pi)$

$$
C_{e}(f, x, \theta) \cap C_{e}(f, x, \phi) \neq \emptyset
$$

for almost every and nearly every $\phi \in(0, \pi)$. ("Almost every and nearly every" means except at most a measure zero set of the first category.)

Proof. Let $B=\left\{B_{n}\right\}$ be a countable basis for the topology of $W$ and $K^{*}$ be the collection of all sets $G$ each of which can be expressed as a finite union of members of $B$. Then $K^{*}$ is also a countable collection of sets $G$. Let $L$ be the exceptional set of the first part of the theorem. Let $x \in L$. Then there is a set $D(x)$ of positive outer measure in $(0, \pi)$ such that for each $\theta \in D(x)$ there is a set $D_{\theta}(x)$ at $x$ in $(0, \pi)$ of positive outer measure such that for each $\phi \in D_{\theta}(x), C_{e}(f, x, \theta) \cap C_{e}(f, x, \phi)=\emptyset$. Thus, for each $\phi$ in $D_{\theta}(x)$ there is a $G_{\phi} \in K^{*}$ with the property that

$$
C_{e}(f, x, \theta) \subset G_{\phi} \quad \text { and } \quad C_{e}(f, x, \phi) \cap \bar{G}_{\phi}=\emptyset .
$$

For $G \in K^{*}$, let $f^{-1}(G)=G^{*}$. Since $K^{*}$ is a countable collection and $M^{*} D_{\theta}(x)>0$, so there is a $G_{\phi}=G_{0}$ such that

$$
C_{e}(f, x, \theta) \subset G_{0} \quad \text { and } \quad C_{e}(f, x, \phi) \cap \bar{G}_{0}=\emptyset
$$


for a set of directions $\phi$ of positive outer measure in $D_{\theta}(x)$. Thus by Lemma 3 , we get $\theta \in \mathcal{V}^{\prime}\left(G_{0}^{*}, x\right)$, where

$$
\mathcal{V}^{\prime}\left(G_{0}^{*}, x\right)=\left\{\theta: \theta \in(0, \pi), \quad d^{*}\left(G_{0}^{*}, x, \theta\right)=1\right\},
$$

and $M^{*} \Pi^{\prime}\left(G_{0}^{*}, x\right)>0$, where

$$
\Pi^{\prime}\left(G_{0}^{*}, x\right)=\left\{\phi: 0<\phi<\pi, \quad d^{*}\left(G_{0}^{*}, x, \phi\right)=0\right\} \subset D_{\theta}(x) .
$$

Thus it is proved that

$$
D(x) \subset \cup\left\{\mathcal{V}^{\prime}\left(G^{*}, x\right): G \in K^{*} \text { and } M^{*} \Pi^{\prime}\left(G^{*}, x\right)>0\right\} .
$$

Since $M^{*} D(x)>0$ there is $G \in K^{*}$ such that $M^{*} \mathcal{V}^{\prime}\left(G^{*}, x\right)>0$ and also $M^{*} \Pi^{\prime}\left(G^{*}, x\right)>0$. These facts together with the measurability of $f$ imply that $M \mathcal{V}\left(G^{*}, x\right)>0$ and $M \Pi\left(G^{*}, x\right)>0$. These facts further imply that $x \in T\left(G^{*}\right)$, where $T\left(G^{*}\right)$ is the set $T(E)$ of Lemma 1 with $E=G^{*}$. Thus, it is finally proved that

$$
L \subset \cup\left\{T\left(G^{*}\right): G \in K^{*}\right\} .
$$

By Lemma 1, each $T\left(G^{*}\right)$ is countable, and since $K^{*}$ is a countable collection so $L$ is a countable set. It completes the proof of the first part.

The proof of the second part can be completed in three more stages, the proof of each stage is almost similar to the proof of the first stage, i.e. proof of the first part, but the term "positive outer measure" is to be replaced by the term "second category" wherever it is needed, and Lemma 1 is to be replaced by Lemma 2 or by Lemma 2.1 or by Lemma 2.2 , conforming to the requirement.

Corollary. Let $f: H \rightarrow W$ be measurable, where $W$ is a compact, normal and second countable topological space. Then except possibly at most a countable set in $\mathbb{R}$, every set $\Pi(x)$ in $(0, \pi)$ of positive outer measure contains a pair of direction $\{\theta, \varphi\}$ such that

$$
C_{e}(f, x, \theta) \cap C_{e}(f, x, \varphi) \neq \emptyset .
$$

If further $f$ is continuous then, except possibly at most a countable set in $\mathbb{R}$, every second category set $\Pi(x)$ in $(0, \pi)$ contains a pair of direction $\{\theta, \varphi\}$ such that

$$
C_{e}(f, x, \theta) \cap C_{e}(f, x, \varphi) \neq \emptyset .
$$

Proof. The proof follows from the theorem. 
Remark. From the definition of functions having restricted Baire property, it appears that the continuous function in our theorem can be replaced by the function having restricted Baire property. This type of extension can be made in several theorems of essential cluster sets for continuous functions.

Question. Does the theorem remain true when

(1) "measurable" is replaced by "arbitrary" and

(2) "continuous" is replaced by measurable"?

\section{References}

[1] C. L. Belna, M. J. Evans, and P. D. Humke, Most directional cluster sets have common values, Fund. Math. 101 (1978), 1-10.

[2] C. Goffman and W. T. Sledd, On essential cluster sets, J. London Math. Soc. 1 (1969), no. 2, 295-302.

[3] V. Jarnik, Sur les fonctions de deux variables reelles, Fund. Math. 27 (1936), 147-150.

[4] A. K. Layek, Intersection of essential cluster sets, Ann. Polon. Math. 41 (1983), 49-55.

[5] A. K. Layek, Directional cluster sets and directional essential cluster sets, Bull. London Math. Soc. 20 (1988), 45-50.

[6] S. N. Mukhopadhyay, On essential cluster sets, Fund. Math. (1973), 160171. 